Editorial

\title{
Periprosthetic joint infection - current scenario on management
}

Keywords: hip arthroplasty, periprosthetic, financial burden

\section{Editorial}

Periprosthetic joint infection (PJI) is one of the complications which hamper the final outcome of joint replacement surgery. This in turn causes increased hospitalization, psychological stress and also need for re-operations which increases financial burden. Even revision surgery will not have much better outcomes in comparison to index surgery. Also there is an increase in mortality rates.

Total knee and total hip replacement are the commonly done procedures. Preventing PJI is the best strategy. PJI has been found to be the most common cause of failure after hip arthroplasty and of early failure after knee replacement. However early and accurate diagnosis helps in effective treatment. There is a combined-approach for diagnosis by utilizing multiple tools together like-radiological, microbiological \&histological. ${ }^{1}$

To diagnose PJI we need to find the cause. Look for the source of infection. Always go for microbial cultures of synovial fluid. This helps in narrowing down causative organism and we will be able to select the antibiotic appropriately. Recent metaanalyses have reported microbiological failure rates of $0-40 \%$ for one- and two-stage revision for infected hip and knee arthroplasties. Failure can be because of patient factors, microbiological factors or errors in diagnosis. In some cases, cultures come as negative. So the recent advancement is that in such cases we can to do next generation sequencing. This has been an effective tool to find the pathogen. ${ }^{2}$

There are strong evidences to say that following tests are supporting to diagnose PJI - such as Serum ESR, CRP, IL-6. Also histopathological tests are of definitive value. As per AAOS recommendation preoperative prophylactic antibiotics should be given before revision surgery. ${ }^{3}$

A systemic disease like rheumatoid arthritis is a risk factor. Patients with RA are having 2-4 times higher risk of periprosthetic joint infection. Other one is diabetes, and in particular DM with insulin treatment, is potential risk for infection. It was four times higher when compared to two-fold increase in those taking oral antidiabetic treatment. Nephropathy, hepatopathy and malignancy in the past medical history are other risk factors. ${ }^{4}$ We need to weigh the risks and benefits of surgery before advising the procedure for patients with these risk factors.
Volume I2 Issue I - 2020

Nithin Sunku
Department of Orthopaedic, RMM Global Hospital \& Trauma
Centre, India

Correspondence: Nithin Sunku, Consultant Orthopaedic \& Joint Replacement Surgeon, RMM Global Hospital \& Trauma Centre, A unit of Global Hospital \& Research Centre, Mt Abu, Abu Road, Rajasthan, India, Email drsnihin@gmail.com

Received: January 30, 2020 | Published: February 17, 2020

Managing periprosthetic joint infections is a challenge to the clinician. There are various classifications available. Also subsequent algorithms of treatment based on such classifications are there. But per say the treatment should be individualized. So many factors play a role and always there should be multidisciplinary approach to decrease the errors in the diagnosis and treatment of these patients.

\section{Acknowledgments}

None.

\section{Conflicts of interest}

The author declares there are no conflicts of interest.

\section{References}

1. Parvizi J, Zmistowski B, Berbari EF, et al. New definition for periprosthetic joint infection: from the workgroup of the musculoskeletal infection society. Clin Orthop Relat Res. 2011;469(11):2992-2994.

2. Goswami K, Parvizi J. Culture-negative periprosthetic joint infection: is there a diagnostic role for next-generation sequencing?, Expert Rev Mol Diagn. 2019:1-4

3. Tubb CC, Polkowksi GG, Krause B. Diagnosis and prevention of periprosthetic joint infections. J Am Acad Orthop Surg. 2020.

4. Breznicky J, Hlavac M, Novak M, et al. Risk factors for periprosthetic joint infection of the hip and knee. Med Glas (Zenica). 2020;17(1). 